\title{
Once-daily pramipexole for the treatment of early and advanced idiopathic Parkinson's disease: implications for patients
}

This article was published in the following Dove Press journal:

Neuropsychiatric Disease and Treatment

I3 May 201 I

Number of times this article has been viewed

\author{
Angelo Antonini' \\ Daniela Calandrella ${ }^{2}$ \\ 'Department for Parkinson's Disease, \\ IRCSS San Camillo, Venice, Italy; \\ 2Department for Parkinson's Disease, \\ Clinic San Pio X, Milan, Italy
}

\begin{abstract}
Immediate-release (IR) pramipexole is indicated for the symptomatic treatment of idiopathic Parkinson's disease (PD), either alone (without levodopa) or in combination with levodopa, that is, during the entire progress of disease up to the advanced stage. It is also currently indicated for the treatment of moderate-to-severe primary restless legs syndrome (RLS). An extended-release (ER) formulation of pramipexole has been developed to allow a once-daily formulation and to provide more stable dopaminergic stimulation. This review summarized the pharmacokinetic profile of pramipexole for both the IR and ER formulations, and discussed the role of pramipexole in the management of early and advanced PD. The introduction of a once-daily formulation of pramipexole poses significant potential advantages for patients and this is reflected by relatively stable plasma levels. The most obvious benefit is convenience of use and better adherence to treatment schedule. Additional advantages may be represented by the opportunity to provide continuous drug delivery in a fashion that could potentially help minimize dyskinesia risk if the drug is used early in the disease course.
\end{abstract}

Keywords: advanced Parkinson's disease, continuous drug delivery, early Parkinson's disease, extended release, immediate release, pramipexole

\section{Introduction}

Pramipexole is a selective dopamine $\mathrm{D}_{2}$ receptor agonist, approved since 1998 in most European countries and in 1997 in the US. Pramipexole is indicated for the symptomatic treatment of idiopathic Parkinson's disease (PD), either alone (without levodopa) or in combination with levodopa, that is, during the entire progress of disease up to the advanced stage. It is also currently indicated for the treatment of moderate-to-severe primary restless legs syndrome (RLS).

Since their introduction, dopamine agonists have become a common first-line treatment for PD and some patients remain on a dopamine agonist through the entire course of their disease. Dopamine agonists are advantageous in several aspects. They do not require carrier-mediated transport in the gut or brain. They act directly on the dopamine receptors without the need for metabolic modification, release, or storage. They also have longer half-lives than levodopa and therefore they produce more persistent dopamine receptor stimulation than levodopa. Their metabolism does not generate free radicals which are considered one of the most important hazards in levodopa treatment, particularly on dopaminergic neurons. ${ }^{1,2}$ This means there is now considerable experience in trials and in clinical practice with dopamine agonists such as pramipexole.
Correspondence: Angelo Antonin Department for Parkinson Disease, IRCCS San Camillo, Viale Alberoni 70 Venice, Italy

Tel $+3904|220755|$

Fax $+3904 \mid 2207469$

Email angelo3000@yahoo.com 
This review summarizes the pharmacokinetic profile of pramipexole for both the immediate release (IR) and extended release (ER) formulations, and discusses the role of pramipexole in the management of early and advanced PD.

Our review of the literature involved performing searches in Medline (1966-February 2011) and the central database in the Cochrane Library (1948-2011) with the specific search terms: 'pramipexole', 'pramipexole extended release', 'mirapexin', 'mirapex', and 'dopamine agonist'. Papers were considered if they were published in the English peerreviewed literature. In addition, reference lists published in review papers were systematically checked for relevant references, regulatory publications issued by the European Committee of Proprietary Medicinal Products and the US Food and Drug Administration were reviewed, and recent releases of information (congress abstracts and press releases) from major PD clinical trials were considered.

\section{Pharmacology}

Pramipexole is a synthetic, nonergot, aminothiazole dopamine agonist with high relative in vitro specificity and full intrinsic activity at the $\mathrm{D}_{2}$ subfamily of dopamine receptors, binding with higher affinity to $\mathrm{D}_{3}$ than to $\mathrm{D}_{2}$ or $\mathrm{D}_{4}$ receptor subtypes. ${ }^{3}$ The relevance of $\mathrm{D}_{3}$ receptor binding in $\mathrm{PD}$ is unknown but it is suggested that the preferential affinity of pramipexole for the $\mathrm{D}_{3}$ receptor subtype could contribute to efficacy in the treatment of psychiatric symptoms of PD-like depression. ${ }^{4}$ Unlike the ergot dopamine agonists, pramipexole has little or no interaction with adrenergic or serotonergic receptors, ${ }^{3}$ which means that pramipexole does not appear to exert any fibrotic effects. ${ }^{5}$

Biochemical and electrophysiological aspects of dopaminergic autoreceptor desensitization have also been described previously. It was observed that sustained pramipexole administration produced desensitization of $\mathrm{D}_{2} / \mathrm{D}_{3}$ and 5-HT1A cell body autoreceptors, as well as a decrease in sensitivity of $\alpha_{2}$-adrenergic cell body autoreceptors. ${ }^{6}$

\section{Pharmacokinetic profile}

Pramipexole has linear pharmacokinetics over its entire therapeutic range. It is rapidly and completely absorbed with peak levels appearing in the bloodstream within 2 hours of oral dosing. It has a high absolute bioavailability of $>90 \%$ and can be administered without regard to meals (time to peak concentration is 3-4 hours with food although the extent of absorption remains unaffected). Pramipexole excretion is primarily renal $(90 \%$ excreted unchanged in the urine) and there is negligible metabolism of pramipexole. ${ }^{7}$
The pharmacokinetic properties of pramipexole at steadystate concentrations were studied in 16 healthy men and women at four dose levels throughout the range recommended for Parkinson's patients. Concentrations of pramipexole were proportional to dose, although the drug's pharmacokinetic properties differed between men and women. The area under the concentration-time curve for each dose level was $35 \%$ to $43 \%$ greater in women, mainly because of a $24 \%$ to $27 \%$ lower oral clearance. The renal clearance of pramipexole accounts for approximately $80 \%$ of oral clearance, and there was a significant correlation between renal and creatinine clearances. The influence of gender could not be distinguished from the influence of age and the resulting reduced creatinine clearance, but the measurement of pharmacokinetic properties produced linear results in both men and women. ${ }^{8}$ Of particular note is the fact that pramipexole undergoes almost no hepatic biotransformation and was found to exert no potent cytochrome P450 (CYP) system inhibition in vitro. ${ }^{9}$

This lack of hepatic metabolism has been suggested to minimize drug-drug interactions, which may be clinically relevant as PD predominantly affects the older generation who are often prescribed multiple medications. ${ }^{10}$

Coadministration of other drugs renally secreted by the cationic transporter system decreased the pramipexole clearance by $9.0 \%$ to $17.7 \% .^{7}$ A pharmacokinetic analysis in Japanese patients demonstrated that coadministration of amantadine decreased the total clearance of pramipexole by $25 \% .{ }^{11}$ Organic cation transporters (OCTs), a family of polyspecific organic cation transporters responsible for the transport of organic cations, including amantadine, are involved in the renal uptake of pramipexole across the basolateral membrane of the proximal tubular epithelial cells in rat. ${ }^{12}$ Amantadine inhibited the uptake of pramipexole by rat OCT1- and rat OCT2-expressing oocytes and rat kidney slices in a concentration-dependent manner, suggesting that an organic cation-specific mechanism is at least partly involved in this drug interaction. ${ }^{12}$

Pramipexole ER is a nonergolinic dopamine receptor agonist available for use as a once-daily oral treatment for the signs and symptoms of early and advanced PD. Continuous dopaminergic stimulation is a therapeutic concept for the development of dopamine agonists with continuous, as opposed to discontinuous or pulsatile, stimulation of striatal dopamine receptors. This concept derived from studies of the normal basal ganglia demonstrating that nigral dopaminergic neurons normally fire continuously and striatal dopamine levels are relatively constant. Once-daily pramipexole ER and three times daily 
pramipexole IR have similar exposure over 24 hours. In a Phase I trial, where pramipexole IR and ER tablets were assessed in fasted state, the minimum and maximum plasma concentration $\left(\mathrm{C}_{\min }, \mathrm{C}_{\max }\right)$ and exposure (AUC) of the same daily dose of pramipexole ER tablets given once daily and pramipexole IR tablets given three times daily were equivalent. Moreover, once-daily administration of pramipexole ER tablets causes a smaller fluctuation in the pramipexole plasma concentration over 24 hours compared to the three times daily administration of pramipexole IR tablets. Maximum plasma concentrations occur at about 6 hours after administration of pramipexole ER tablets (Figure 1) and a steady state of exposure is reached after 5 days of continuous dosing at the latest. ${ }^{13}$

A level A in vitro/in vivo correlation (IVIVC) was established and evaluated for the pramipexole ER formulations. ${ }^{14}$ Pharmacokinetic profiles for ER formulations were described based on the in vitro release model and the population pharmacokinetic model developed from the IR formulation. This IVIVC also showed that pramipexole of the ER formula was steadily absorbed all through the entire intestine including the colon. A pharmacokinetic-pharmacodynamic study using in vivo microdialysis in rats showed, in agreement with in vivo microdialysis measurements, a continuous decrease of extracellular dopamine levels and a continuous pramipexole exposure in rats with continuous release of pramipexole maintained by subcutaneous implantation of minipumps. In contrast, pramipexole IR $(0.3 \mathrm{mg} / \mathrm{kg})$ produced a transient

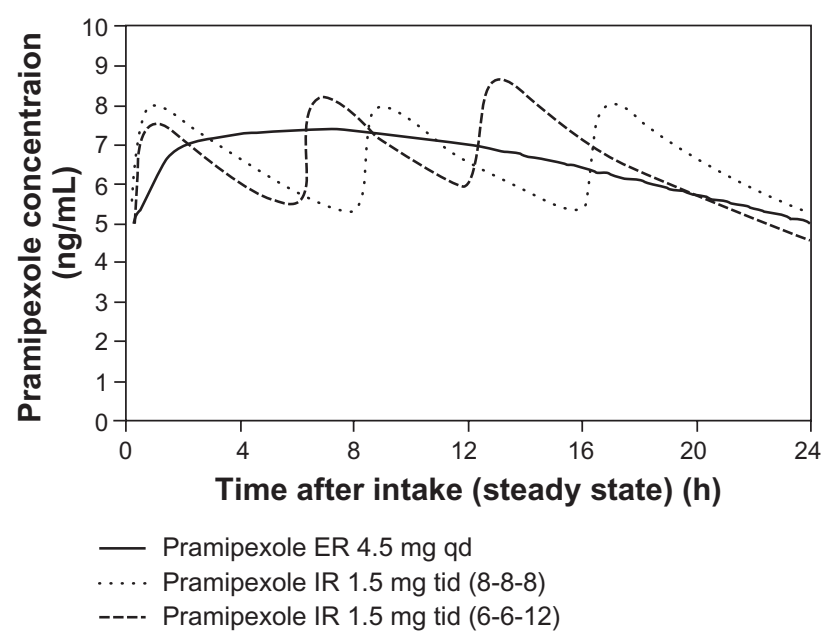

Figure I Simulated concentration-time profile of pramipexole ER and pramipexole IR at steady state in Parkinson patients after intake of pramipexole ER $4.5 \mathrm{mg}$ qd or pramipexole IR tid in intervals of 8 hours (8-8-8) or different intervals of 6 and 12 hours $(6-6-12)$.

Notes: Reproduced with permission from Eisenreich W, Sommer B, Hartter S, Jost WH. Pramipexole extended release: a novel treatment option in Parkinson's disease. Parkinsons Dis. 2010;2010:612-619.16

Abbreviations: ER, extended release; IR, immediate release; tid, three times daily; qd, once daily. decrease of extracellular dopamine levels over 6 hours and showed maximum pramipexole levels 2 hours after dosing which decreased over the following 6-8 hours. ${ }^{15}$

The variability among other individuals in this process was rather low and not influenced by other factors such as food. ${ }^{16}$

\section{Therapeutic efficacy of pramipexole ER in early PD}

Clinical trials of IR pramipexole have included over 1,200 patients in the early stages of PD (disease durations up to 2.4 years), with 698 patients receiving pramipexole for up to 4 years and maintenance dose in all but one of the studies standardized between 0.375 and $4.5 \mathrm{mg}$ of salt per day. ${ }^{17-21}$

These studies have consistently shown pramipexole efficacy in treating the symptoms of early PD with significantly different changes versus placebo in Unified Parkinson's Disease Rating Scale (UPDRS) scores of 2-3 points in UPDRS Part II (activities of daily living [ADL]), 3-5 points in UPDRS Part III (motor) scores and 5-6.5 points in Total UPDRS scores. ${ }^{17,18,20}$ Thus, even in the early stages of the disease, where baseline UPDRS scores are lower, pramipexole provides clinically relevant efficacy. ${ }^{22}$

A randomized, double-blind, placebo and active comparator-controlled trial on ER pramipexole in the early stage of PD was carried out by Hauser et al. ${ }^{23}$ In this study, 259 patients were randomized $(2: 2: 1)$ to double-blind pramipexole ER (0.375-4.5 mg, once daily), IR (0.125$1.5 \mathrm{mg}$ tid), or placebo. Patients were flexibly titrated to an effective dose over 7 weeks, then maintained for an additional 26 weeks. A preplanned interim analysis at week 18 confirmed superiority of pramipexole ER over placebo, and in the final analysis at week 33 , noninferiority was demonstrated between the two pramipexole formulations with an adjusted mean change in UPDRS II + III score of -8.6 points for ER $(\mathrm{n}=213)$ and -8.8 points for IR $(\mathrm{n}=207)$. The incidence and severity of side effects did not differ either.

Another study ${ }^{24}$ in early PD patients was carried out on 259 early PD patients. This controlled, double-blind trial (ER pramipexole:IR:placebo, 2:2:1) also focused on the effect by employing UPDRS II and III plus Clinical Global Impression - Improvement (CGI-I) and Patient Global Impression - Improvement (PGI-I). An improvement by 7.5 points was found in the group treated with IR pramipexole $(\mathrm{n}=99)$. The group taking ER pramipexole $(\mathrm{n}=99)$ scored 7.4 points and the placebo group $(n=42)$ scored 2.7 points. Response rates were determined by GCI-I and PGI-I and were indicative of improvements for ER pramipexole at 37\% and $35.6 \%$, for IR pramipexole at $48 \%$ and $23.8 \%$, and for 
placebo at $18 \%$ and $12 \%$ in weeks 18 and 33 , respectively. The authors concluded that both pramipexole formulas were safe and superior to placebo, aside from being well tolerated, the efficacy of ER was similar to IR with comparable adverse effects.

\section{Therapeutic efficacy of pramipexole ER in advanced PD}

Several randomized placebo-controlled trials have assessed the efficacy of pramipexole IR as adjunct to levodopa in patients suffering from levodopa motor fluctuations. ${ }^{25-31}$ These studies consistently showed significant improvements of UPDRS motor and ADL scores versus placebo and Total UPDRS scores were improved by $20 \%-40 \%$ versus baseline (compared with 4\%-13\% with placebo). At the same time, the introduction of pramipexole adjunct therapy meant that patients could reduce their levodopa dose by an average of $30 \%$. Retrospective analyses of randomized, placebocontrolled trials with pramipexole, pergolide, ropinirole, tolcapone, or entacapone as adjuvant therapies to levodopa revealed that the reduction in levodopa dose was most significant for pramipexole and entacapone $(P<0.0001)$. In addition, the most significant reduction in OFF duration was with pramipexole (approximately 30\% reduction) and entacapone $(P<0.001){ }^{32}$

The most significant study on ER pramipexole in the advanced stage of Parkinson disease was carried out by Schapira et al. ${ }^{33}$ In this study, patients with advanced PD taking levodopa plus a dopa decarboxylase inhibitor were randomized (1:1:1) to pramipexole ER (0.375-4.5 mg qd), or IR (0.125-1.5 mg tid), or placebo. Patients were flexibly titrated to an effective dose over 7 weeks, then maintained for an additional 26 weeks. The adjusted mean change in UPDRS II + III at Week 18 was - 11.0 points for pramipexole ER, -12.6 points for pramipexole IR, and -6.3 points for placebo $(P<0.0002$ and $P<0.0001$ vs placebo, respectively). The adjusted mean change in percentage OFFtime was -13.3 for pramipexole $E R,-15.7$ for pramipexole IR, and -9.0 for placebo $(P=0.0174$ and $P<0.0001$ vs placebo, respectively), corresponding to an absolute placebocorrected improvement of -0.5 hour for pramipexole ER and -1.0 hour for pramipexole IR.

\section{Dosage and administration}

Pramipexole is typically initiated at a dose of $0.25 \mathrm{mg}$ and gradually titrated to $1.5 \mathrm{mg}$ tid depending on patient response. It should be noted that in dosing studies, the group receiving $0.5 \mathrm{mg}$ tid had benefits comparable with those receiving higher doses, but with fewer side effects. It is therefore suggested to titrate patients to $0.5 \mathrm{mg}$ tid and use higher doses on an individual basis rather than as part of a routine titration schedule. Pramipexole ER tablets should be swallowed whole with water, and must not be chewed, divided, or crushed. The tablets may be taken either with or without food and should be taken each day at about the same time. Doses of pramipexole ER should be increased gradually from a starting dose of $0.26 \mathrm{mg}$ of base $(0.375 \mathrm{mg}$ of salt) per day and then increased as needed every 5-7 days up to a daily dose of $1.05 \mathrm{mg}$ of base (1.5 mg of salt). If a further dose increase is necessary the daily dose should be increased by $0.52 \mathrm{mg}$ of base $(0.75 \mathrm{mg}$ of salt) at weekly intervals up to a maximum dose of $3.15 \mathrm{mg}$ of base ( $4.5 \mathrm{mg}$ of salt) per day. Studies have shown that patients already taking pramipexole tablets may be switched to pramipexole ER tablets overnight, at the same daily dose. ${ }^{34}$ After switching to pramipexole ER tablets, the dose may be adjusted depending on the patient's therapeutic response.

A second switch study was conducted in Japan by Mizuno et $\mathrm{al}^{35}$ also making use of the overnight approach. This double-blind study was run for 12 weeks in 112 patients, followed by a 4-week open phase. The efficacy was assessed by UPDRS parts II and III. Differences in UPDRS rating were irrelevant (IR pramipexole 13.3, ER 13.6). The switch was successful in $83 \%$ of patients. The rate of adverse effects did not differ.

\section{Conclusion}

Current evidence favors therapy initiation in PD with a dopamine agonist, such as pramipexole, except for elderly patients or those presenting cognitive abnormalities at onset. However, levodopa should not be withheld from patients in whom adequate symptomatic control cannot be otherwise obtained. The choice of initial therapy will depend on the individuals need for symptomatic efficacy, the degree of motor impairment, and the risks of adverse effects.

In more advanced disease, when levodopa therapy is complicated by the emergence of wearing-off and other complications, a dopamine agonist (such as pramipexole) may be initiated or the current dose increased to reduce severity of OFF periods and to allow reduction of levodopa doses.

In this respect the recently introduced pramipexole ER formulation may present significant advantages in terms ensuring an improved adherence to treatment schedule. Compared to the IR formulation, the substance itself is unchanged, meaning there is an identical receptor 
profile, identical efficacy, and identical receptor binding. The half-life is also the same, but the continuous release results in an overall prolonged plasma half-life. The potential advantages include maintaining more consistent dopaminergic activity with steadier plasma levels, increased tolerability, greater compliance from a simpler once-daily dosing regimen and ease in dose titration. In addition, continuous delivery of dopaminergic agents is believed to more closely mimic the physiological tone of the nigrostriatal dopamine system as opposed to pulsatile delivery of multiple daily doses.

ER pramipexole can be used in both early and late PD. A switch can take place overnight, with the ratio being 1:1. In most cases, the patient is not going to be affected by this switch. More efficaciousness, increased dyskinesia, or undesired effects are not to be expected. In a few cases, the effect may be less, which would require a dose adjustment.

\section{Disclosure}

The authors declare no conflict of interest in this work.

\section{References}

1. Olanow CW, Stern MB, Sethi K. The scientific and clinical basis for the treatment of Parkinson disease. Neurology. 2009;72(21 Suppl 4): S1-S136.

2. Horstink M, Tolosa E, Bonuccelli U, et al. Review of the therapeutic management of Parkinson's disease. Report of a joint task force of the European Federation of Neurological Societies and the Movement Disorder Society-European Section. Part I: early (uncomplicated) Parkinson's disease. Eur J Neurol. 2006;13(11):1170-1185.

3. Piercey MF. Pharmacology of pramipexole, a dopamine D3-preferring agonist useful in treating Parkinson's disease. Clin Neuropharmacol. 1998;21(3):141-151.

4. Maj J, Rogoi Z, Margas W, Kata M, Dziedzicka-Wasylewska M. The effect of repeated treatment with pramipexole on the central dopamine D3 system. J Neural Transm. 2000;107(12):1369-1379.

5. Kvernmo T, Härtter S, Burger E. A review of the receptor-binding and pharmacokinetic properties of dopamine agonists. Clin Ther. 2006; 28(8):1065-1078.

6. Chernoloz O, El Mansari M, Blier P. Sustained administration of pramipexole modifies the spontaneous firing of dopamine, norepinephrine, and serotonin neurons in the rat brain. Neuropsychopharmacology. 2009;34(3):651-661

7. Wright CE, Sisson TL, Ichhpurani AK, Peters GR. Steady-state pharmacokinetic properties of pramipexole in healthy volunteers. J Clin Pharmacol. 1997;37(6):520-525.

8. Deleu D, Northway MG, Hanssens Y. Clinical pharmacokinetic and pharmacodynamic properties of drugs used in the treatment of Parkinson's disease. Clin Pharmacokinet. 2002;41(4):261-309.

9. Wynalda MA, Wienkers LC. Assessment of potential interactions between dopamine receptor agonists and various human cytochrome P450 enzymes using a simple in vitro inhibition screen. Drug Metab Dispos. 1997;25(10):1211-1214.

10. Hubble JP. Pre-clinical studies of pramipexole: clinical relevance. Eur J Neurol. 2000;7(Suppl 1):15-20.

11. Wu MJ, Ing TS, Soung LS, Daugirdas JT, Hano JE, Gandhi VC. Amantadine hydrochloride pharmacokinetics in patients with impaired renal function. Clin Nephrol. 1982;17(1):19-23.
12. Ishiguro N, Saito A, Yokoyama K, Morikawa M, Igarashi T, Tamai I. Transport of the dopamine D2 agonist pramipexole by rat organic cation transporters OCT1 and OCT2 in kidney. Drug Metab Dispos. 2005; 33(4):495-499.

13. Jenner P, Konen-Bergmann M, Schepers C, Haertter S. Pharmacokinetics of a once-daily extended-release formulation of pramipexole in healthy male volunteers: three studies. Clin Ther. 2009;31(11):2698-2711.

14. Soto E, Haertter S, Koenen-Bergmann M, Staab A, Trocóniz IF Population in vitro-in vivo correlation model for pramipexole slowrelease oral formulations. Pharm Res. 2010;27(2):340-349.

15. Ferger B, Buck K, Shimasaki M, Koros E, Voehringer P, Buerger E. Continuous dopaminergic stimulation by pramipexole is effective to treat early morning akinesia in animal models of Parkinson's disease: A pharmacokinetic-pharmacodynamic study using in vivo microdialysis in rats. Synapse. 2010;64(7):533-541.

16. Eisenreich W, Sommer B, Hartter S, Jost WH. Pramipexole extended release: a novel treatment option in Parkinson's disease. Parkinsons Dis. 2010;2010:612-619.

17. Hubble JP, Koller WC, Cutler NR, et al. Pramipexole in patients with early Parkinson's disease. Clin Neuropharmacol. 1995;18(4):338-347.

18. Shannon KM, Bennett JP Jr, Friedman JH. Efficacy of pramipexole, a novel dopamine agonist, as monotherapy in mild to moderate Parkinson's disease. The Pramipexole Study Group. Neurology. 1997; 49(3):724-728.

19. Parkinson Study Group. Pramipexole vs levodopa as initial treatment for Parkinson disease: A randomized controlled trial. Parkinson Study Group. JAMA. 2000;284(15):1931-1938.

20. Safety and efficacy of pramipexole in early Parkinson disease. A randomized dose1 ranging study. Parkinson Study Group. JAMA. 1997;278(2):125-130.

21. Holloway RG, Shoulson I, Fahn S, et al. Pramipexole vs levodopa as initial treatment for Parkinson disease: a 4-year randomized controlled trial. Arch Neurol. 2004;61(7):1044-1053.

22. Schrag A, Sampaio C, Counsell N, Poewe W. Minimal clinically important change on unified Parkinson's disease rating scale. Mov Disord. 2006;21(8):1200-1207.

23. Hauser RA, Schapira AH, Rascol O, et al. Randomized, double-blind, multicenter evaluation of pramipexole extended release once daily in early Parkinson's disease. Mov Disord. 2010;25(15):2542-2549.

24. Hauser RA, Salin L, Koester J. Double-blind evaluation of pramipexole extended release (ER) in early Parkinson's disease. Neurology. 2009;72(11 Suppl 3):A412-A413.

25. Lieberman A, Ranhosky A, Korts D. Clinical evaluation of pramipexole in advanced Parkinson's disease: results of a double-blind, placebocontrolled, parallel-group study. Neurology. 1997;49:162-168.

26. Guttman M. Double-blind comparison of pramipexole and bromocriptine treatment with placebo in advanced Parkinson's disease. International Pramipexole-Bromocriptine Study Group. Neurology. 1997;49(4):1060-1065.

27. Wermuth L. A double-blind, placebo-controlled, randomized, multicenter study of pramipexole in advanced Parkinson's disease. Eur J Neurol. 1998;5(3):235-242.

28. Pinter MM, Pogarell O, Oertel WH. Efficacy, safety, and tolerance of the non-ergoline dopamine agonist pramipexole in the treatment of advanced Parkinson's disease: a double blind, placebo controlled, randomised, multicentre study. J Neurol Neurosurg Psychiatry. 1999; 66(4):436-441.

29. Mizuno Y, Yanagisawa N, Kuno S, et al. Randomized, double-blind study of pramipexole with placebo and bromocriptine in advanced Parkinson's disease. Mov Disord. 2003;18(10):1149-1156.

30. Weiner WJ, Factor SA, Jankovic J, et al. The long-term safety and efficacy of pramipexole in advanced Parkinson's disease. Parkinsonism Relat Disord. 2001;7(2):115-120.

31. Moller JC, Oertel WH, Koster J, Pezzoli G, Provinciali L. Long-term efficacy and safety of pramipexole in advanced Parkinson's disease: results from a European multicenter trial. Mov Disord. 2005;20(5): $602-610$. 
32. Inzelberg R, Carasso RL, Schechtman E, Nisipeanu P. A comparison of dopamine agonists and catechol- $O$-methyltransferase inhibitors in Parkinson's disease. Clin Neuropharmacol. 2000;23(5):262-266.

33. Schapira AH, Barone P, Hauser R, et al. Efficacy and safety of pramipexole extended-release for advanced Parkinson's disease [abstract]. Mov Disord. 2009;24(Suppl 1):S277.

34. Rascol O, Barone P, Hauser RA, et al; Pramipexole Switch Study Group. Efficacy, safety, and tolerability of overnight switching from immediate- to once daily extended-release pramipexole in early Parkinson's disease. Mov Disord. 2010;25(14):2326-2332.
35. Mizuno Y, Yamamoto M, Kuno S, Hasegawa K, Hattori N, Kagimura T. Efficacy of pramipexole extended release (ER) and switching from pramipexole immediate release (IR) to ER in Japanese advanced Parkinson's disease (PD) patients. Proceedings of the 18th WFN World Congress on Parkinson's Disease and Related Disorders, Miami Beach, FL, USA; 2009:Poster 2.192.

\section{Publish your work in this journal}

Neuropsychiatric Disease and Treatment is an international, peerreviewed journal of clinical therapeutics and pharmacology focusing on concise rapid reporting of clinical or pre-clinical studies on a range of neuropsychiatric and neurological disorders. This journal is indexed on PubMed Central, the 'PsycINFO' database and CAS, and is the official journal of The International Neuropsychiatric Association (INA). The manuscript management system is completely online and includes a very quick and fair peer-review system, which is all easy to use. Visit http://www.dovepress.com/testimonials.php to read real quotes from published authors.

Submit your manuscript here: http://www.dovepress.com/neuropsychiatric-disease-and-treatment-journal 